\title{
DEFINIÇÃO DA ENERGIA LIVRE DE HELMHOLTZ EM FONTES DIDÁTICAS
}

\author{
DEFINICIÓN DE ENERGÍA LIBRE DE HELMHOLTZ EN FUENTES \\ DIDÁCTICAS
}

DEFINITION OF HELMHOLTZ FREE ENERGY IN DIDACTIC SOURCES

DOI: $10.22481 /$ rbba.v8i2.5784

\begin{abstract}
Jornandes Jesús Correia
Universidade Estadual do Sudoeste da Bahia, Brasil

ORCID: https://orcid.org/0000-0002-0035-2300

Lattes: http://lattes.cnpq.br/5594644574555987

Endereço eletrônico: jornandes.correia@uesb.edu.br

Gabriel Fonseca Guimarães

Universidade Estadual do Sudoeste da Bahia, Brasil

ORCID: https://orcid.org/0000-0002-8795-897X

Lattes: http://lattes.cnpq.br/6850946554302469

Endereço eletrônico: gabrielfonseca1415@gmail.com
\end{abstract}

\begin{abstract}
RESUMO
Este artigo tem como objetivo analisar em fontes didáticas: a definição da Energia Livre de Helmholtz, definindo e discutindo a variação da sua intensidade; discutir a sua conservação e as condições do seu valor. A Categoria de Análise está referendada nas abordagens histórica e postulatória das fontes pesquisadas, balizada pelo Silogismo de Aristóteles. Devido à falta de sintonia das definições apresentadas nas fontes pesquisadas, apresenta uma definição para o Potencial de Helmholtz, bem como uma análise das condições da sua variação e da sua conservação. Observa-se que a maioria das fontes analisadas se limita a fazer a leitura da relação matemática que a representa, em detrimento de uma abordagem epistemológica. $\mathrm{O}$ artigo também busca definir a grandeza potencial de grande complexidade representada pela Energia Livre de Helmholtz como constitutiva, ainda, de um grande desafio.
\end{abstract}


Palavras-chave: Energia Livre de Helmholtz; Ensino da Termodinâmica; Funções Potenciais; Livros didáticos.

\title{
RESUMEN
}

Este artículo tiene como objetivo analizar en fuentes didácticas: la definición de la energía libre de Helmholtz, definiendo y discutiendo la variación de su intensidad; discutir su conservación y las condiciones de su valor. La categoría de análisis está respaldada por los enfoques históricos y postulatorios de las fuentes investigadas, marcados por el silogismo de Aristóteles. Debido a la falta de ajuste de las definiciones presentadas en las fuentes investigadas, presenta una definición para el potencial de Helmholtz, así como un análisis de las condiciones de su variación y su conservación. Se observa que la mayoría de las fuentes analizadas se limitan a leer la relación matemática que la representa, en detrimento de un enfoque epistemológico. El artículo también busca definir la grandeza potencial de gran complejidad representada por la Energía Libre de Helmholtz como un gran desafío.

Palabras clave: Energía libre de Helmholtz; Enseñanza de la termodinámica; Funciones potenciales; Libros didácticos.

\begin{abstract}
This article aims to analyze in didactic sources: the definition of Helmholtz Free Energy, defining and discussing the variation of its intensity; discuss its conservation and the conditions of its value. The Analysis Category is supported by the historical and postulatory approaches of the researched sources, marked by Aristotle's Syllogism. Due to the lack of tuning of the definitions presented in the researched sources, it presents a definition for the Helmholtz Potential, as well as an analysis of the conditions of its variation and its conservation. It is observed that most of the sources analyzed are limited to reading the mathematical relationship that represents it, to the detriment of an epistemological approach. The article also seeks to define the potential greatness of great complexity represented by the Free Energy of Helmholtz as constituting, still, a great challenge.
\end{abstract}

Keywords: Helmholtz Free Energy; Teaching of Thermodynamics; Potential Functions; Didactic Books.

\section{INTRODUÇÃO}

Apesar da Energia Livre de Helmholtz, também denominada por Função de Helmholtz ou Potencial de Helmholtz, ser de fundamental importância para a determinação da energia a ser obtida de um sistema e ser de grande relevância ao estudo de sistemas em equilíbrio 
termodinâmico à volume constante, a sua definição ainda é pouco clara mesmo entre os estudiosos da área. Isso se deve ao fato da função de Helmholtz se tratar de uma grandeza potencial, o que certamente dificulta a sua definição. Outro motivo que pode justificar tais faltas de clareza é que essas mesmas dificuldades de definição também se aplicam à Energia Interna, à Temperatura e à Entropia, que também compõem a equação que descreve o Potencial de Helmholtz.

Percebemos, por uma amostragem, que tanto alguns livros didáticos quanto alguns professores costumam ter dificuldade quando se propõem a discutir o significado da Energia Livre de Helmholtz. Mesmo quando pesquisamos em fóruns conceituados, destinados a debates acadêmicos, como o Research Gate, no qual apenas professores podem participar, ainda nos deparamos com perguntas como a do professor Vahid Ebrahimzade Isfahani, do Institute of Energy and Climate Research 2:

Minha pergunta é sobre Energia Livre de Helmholtz que é definida como:

$F=U-T S$

onde $U$ é a energia interna, $T$ é a temperatura e $\mathrm{S}$ é a entropia. Eu procurei em alguma literatura para descobrir por que ela é chamada de "livre". O principal raciocínio é que "TS" é a energia térmica necessária para que nosso sistema com a temperatura $T$ e a entropia $S$ possa ser criado. Se subtrairmos este termo da energia interna, teríamos todo tipo de energia que poderia ser extraída como trabalho.

Eu raciocinei comigo mesmo que "TS" é obtido a partir da relação "Delta $Q=T$ Delta $S$ ". Aqui $S_{0}$ é o $S$ inicial em "Delta $S$ ", que é a entropia na temperatura zero.

Delta $Q=T\left(S-S_{0}\right) \Rightarrow$ Delta $Q-T S$ (a energia térmica).

Essa relação é apenas para processos reversíveis onde não há dissipação. Mas, na realidade, não temos processos reversíveis que questionem a explicação no primeiro parágrafo.

O que eu quero saber é se a primeira explicação dada é correta ou não.

Minha segunda pergunta: alguém pode justificar uso do termo energia livre?

Por que é definido como equação (1)? É como uma transformação de Legendre para definir uma forma alternativa de energia interna não em termos de S, mas T? (2013)

Podemos inferir, a partir da provocação do professor Isfahani, que os livros abordam pouco sobre a definição da Energia Livre de Helmholtz ou não são claros na sua abordagem, ao ponto de um pesquisador da área ainda apresentar dificuldade para compreendê-la. E isso deixa claro que uma pesquisa voltada para a definição da Energia Livre de Helmholtz se faz extremamente necessária, uma vez que podemos estar contribuindo para que um leitor amplie sua compreensão sobre definições de grandezas físicas. 
Percebemos, também, pelos questionamentos do professor Isfahani, que apesar de ele demonstrar ter estudado e compreendido o tema, ele ainda apresenta dúvidas. Como exemplo, podemos citar o comentário dele sobre a equação da Segunda Lei da Termodinâmica "Delta $Q$ - TS", apresentando-a como uma variação do calor, o que não faz sentido físico, pois, calor é uma Função de Transformação e não uma grandeza Potencial. Logo, não deveria abordar variação de Calor, porque Calor não é uma grandeza pontual e sim de transformação. Correia e Magalhães, sobre Obstáculos Epistemológicos e o Conceito de Calor (2009), debruçam sobre as dificuldades enfrentadas para se definir Calor e ainda apresentam os motivos históricos que visam contrapor à notação do doutor Isfahani, mesmo que o "tratamento" matemático sugere o uso do delta maiúsculo antes do $Q$.

Após essa provocação no site Research Gate, a discussão continua, em que cada professor apresenta uma definição diferente para uma mesma grandeza. Essa mesma problemática também foi encontrada nos livros pesquisados. Além da não existência de uma definição clara, percebe-se uma grande dissonância entre as fontes, em que cada fonte apresenta uma definição com pouca ligação com as outras.

A equação apresentada pelo professor Isfahani para representar a Segunda Lei da Termodinâmica traduz uma dificuldade na definição de grandezas, que não é uma exclusividade inerente da Energia Livre de Helmholtz.

Nesse sentido, o Grupo de Estudos e Pesquisas em Didática das Ciências Experimentais e da Matemática (GDICEM), vinculado ao Museu Pedagógico Casa Padre Palmeira (MP), vem investigando definições em fontes didáticas tanto das Funções de Estado da Termodinâmica, quanto das Funções de Transformações.

Esta linha de pesquisa do GDICEM iniciou suas atividades em 2008 com o Projeto de Pesquisa intitulado "Os conceitos em livros didáticos de Física - problemas relacionados as definições de fenômenos físicos", submetido ao Departamento de Ciências Exatas e Tecnológicas (DCET), Campus de Vitória da Conquista, Bahia, da Universidade Estadual do Sudoeste da Bahia (UESB) e cadastrado junto à Gerência de Pesquisa desta Universidade. A partir da pesquisa em fontes didáticas, já foram publicados os seguintes artigos: As Definições de Calor (CORREIA; MAGALHÃES; LIMA, 2008) e (CORREIA; MAGALHÃES, 2009); A Definição de Trabalho de uma Força (CORREIA; JOSÉ, 2011); A Definição de Entropia (CORREIA; JOSÉ, 2013); A Conservação de Energia (CORREIA; ORTIGOZA, 2015); a 
Definição de Temperatura (CORREIA, 2017); a Definição de Energia Interna (CORREIA; OLIVEIRA, 2018); e a Definição de Entalpia (CORREIA; OLIVEIRA, 2019).

Esses artigos reforçam a hipótese que as grandezas relacionadas à Termodinâmica não estão bem definidas na maioria das fontes pesquisadas.

Leite e Pinho (2001) abordam sobre essa dificuldade na apresentação de definições:

É incontroverso que a interpretação de um dado discurso escrito ou oral só pode ser correcta tendo em consideração o contexto em que as expressões são usadas, o que é tanto mais importante quanto é certo que os mesmos vocábulos podem ser usados em diversos contextos com significados diferentes. Muitas palavras do vocabulário corrente são usadas em Física com um significado preciso e bem definido, e frequentemente os alunos, familiarizados com o sentido comum dessas palavras, não se apercebem do seu significado específico no contexto da Física. Por outro lado, os professores de Física do Ensino Superior têm como um dado adquirido o sentido específico desses termos e usam-nos naturalmente, sem se preocuparem em defini-lo ou clarificá-lo no contexto da disciplina.

O resultado é muitas vezes os estudantes pensarem que entenderam o que de facto não entenderam ou construírem, com base nos seus conhecimentos prévios e no sentido que atribuem às palavras, teorias incorrectas, não científicas, ou apreensões incorrectas de conceitos físicos. Grandezas definidas em ciência de um determinado modo podem, por isso, ser mal interpretadas no sentido de significarem qualquer coisa diferente (LEITE; PINHO, 2001, p.459).

E isso fica evidente nas afirmativas "os mesmos vocabulários podem ser usados em diversos contextos com significados diferentes" e "O resultado é muitas vezes os estudantes pensarem que entenderam o que de facto não entenderam ou construírem”. O que na Física é extremamente problemático, pois, quando não conseguimos dar significado ao que estamos abordando, nos tornamos reféns da repetição de equações matemáticas sem conseguir, assim, “dar asas à imaginação" para conjecturar um novo método de solução para um problema proposto ou sem conseguir interpretar como poderia ser aplicado na prática.

Sendo assim, existe a necessidade de definir melhor essas grandezas físicas, a exemplo de Gilbert (1982), ao afirmar que muitos cientistas possuem um entendimento intuitivo, porém não conseguem definir as grandezas de maneira que as tornem claras. Por outro lado, sem o empenho desses pesquisadores, a compreensão de fenômenos ou comportamentos físicos estaria mais dificultada. Ou seja, temos diversos textos que abordam sobre a Energia Livre de Helmholtz e um dos nossos objetivos aqui é destacar qual a definição apresentada está mais clara. 
Este artigo encontra-se estruturado da seguinte forma: na seção 2 apresentamos uma discussão sobre a definição para Energia Livre de Helmholtz. Na seção 3 mostramos a relação matemática da Função de Helmholtz e discutimos equação que trata da sua variação. Na seção 4 descrevemos a estrutura com a qual os textos didáticos foram analisados, fundamentada nas categorias histórica e postulatória, balizados pelo Silogismo de Aristóteles. Na seção 5 apresentamos a nossa análise dos textos. Finalmente, na seção 6, apresentamos as categorias das abordagens das fontes pesquisadas.

\section{A definição de Energia Livre de Helmholtz}

A dificuldade em definir Energia Livre de Helmholtz é atribuída à condição dela ser uma função potencial. O desafio torna ainda maior quando se busca, além da sua definição, a definição da sua variação, o significado do seu máximo e do seu mínimo, bem como a conservação dessa função. Para tanto, vale a pena salientar que a discussão que ora fazemos é para sistemas fechados. Além disso, também não faremos considerações em nível microscópico, porque, até então, estamos nos limitando ao campo da Termodinâmica Clássica.

Para a real compreensão de uma grandeza física, acreditamos que seja necessário defini-la e apresentar a interpretação da sua relação matemática, conforme salientam Correia e Oliveira (2019), quando afirmam "nessa perspectiva, a leitura da relação matemática que representa uma grandeza física deverá descrever um fenômeno da natureza" $(2019$, p.330). Ou seja, quando analisamos uma grandeza física, devemos descrever o fenômeno da natureza ao qual ela está associada.

A função de Helmholtz $(F)$, representada matematicamente pela equação $F=U-T S$, ainda nos desafia quanto à sua interpretação, uma vez que cada uma das grandezas $U, T, S$ precisa ser definida separadamente, bem como interpretar e definir o produto TS.

A grandeza “ $U$ ", que representa a Energia Interna de um sistema, é toda energia que um sistema possui devido à sua constituição interna e foi assim definida por Correia e Oliveira (2019, p.331):

Da Primeira Lei da Termodinâmica, já é sabido que existe uma Função de Estado $U$ associada ao sistema, cuja variação é dada pelo saldo das trocas de energia desse sistema ao interagir com outro sistema. Essa função é denominada por Energia Interna, que representa toda energia de um Sistema Termodinâmico devido à sua constituição interna.

\begin{tabular}{l|l} 
Revista RBBA & Revista Binacional Brasil Argentina
\end{tabular} 
Todavia, sabemos que quando um sistema dotado de uma Energia Interna $(U)$ é destruído, não podemos recuperar toda a Energia Interna desse sistema. Entretanto, a Primeira Lei da Termodinâmica garante o Princípio da Conservação da Energia. Logo, a energia total de um sistema isolado deve permanecer constante. Como estamos tratando de sistemas fechados, essa energia não pode simplesmente desaparecer desse sistema. A Entropia $(S)$, grandeza que limita a quantidade da energia a ser disponibilizada, foi definida por Máximo e Alvarenga (1977, p.650):

Suponha que uma certa massa de água quente seja misturada com uma porção de água fria. Como sabemos, este sistema, resultante da mistura, termina por alcançar uma mistura de equilíbrio, que tem o mesmo valor em qualquer ponto do sistema.

Evidentemente, antes de ser efetuada a mistura, teria sido possível fazer uma máquina térmica operar usando as massas de água mencionadas como fonte quente e fria dessa máquina. Isto é, a energia que foi transferida da massa de água quente para a fria poderia ter sido utilizada para realizar trabalho (energia útil).

Entretanto, após a mistura, sendo atingida a uniformidade de temperatura do sistema, embora não tenha havido desaparecimento de energia, não é mais possível convertê-la em trabalho. Vemos então que uma parte da energia tornou-se indisponível, em outras palavras, não podemos usá-la de forma útil. Para aquela parte de energia continuasse disponível para a realização de trabalho, seria necessário que o sistema (suposto isolado) voltasse espontaneamente às condições iniciais, isto é, a mistura se separasse nas duas porções quente e fria primitivas. De nossa experiência diária sabemos que isto nunca ocorre, ou seja, o processo que levou à homogeneização da temperatura é irreversível.

Outra maneira de analisar este processo consiste em observar que o sistema inicialmente encontrava-se em condição mais organizada, isto é, de maior ordem, com as moléculas de maior energia cinética média (água quente) separadas das moléculas de menor energia cinética (água fria). Depois que ocorre a mistura, o sistema torna-se mais desordenado, com as moléculas distribuídas aleatoriamente, havendo uma uniformidade da temperatura.

Esta citação acima introduz a ideia da Entropia, decorrente da impossibilidade de transferência da Energia Térmica (Energia Útil), devido ao Equilíbrio Térmico entre os dois sistemas. Nesse sentido, a Variação da Entropia indica que a energia útil está degradando, mas não é a medida da energia degradada.

Essa forma de tratar "energia útil", apresentada pela definição de Entropia, é extremamente importante para dar significado à Energia Livre de Helmholtz.

\begin{tabular}{l|l} 
Revista RBBA & Revista Binacional Brasil Argentina
\end{tabular} 
Já a Temperatura, Costa a abordou da seguinte forma: "A temperatura é o conceito físico que nos permite medir o estado térmico de um sistema, estabelecendo a sua maior ou menor capacidade de transmitir calor, ou ainda, de acordo com a teoria cinética, a energia cinética média de suas moléculas” (1971, p.35). Entretanto, Correia (2017, p.211) salienta:

\begin{abstract}
Ainda cabe comentar que não existe "transmissão de Calor" e que a capacidade de transmitir energia térmica não está na Temperatura de um sistema, e sim na diferença de Temperatura entre dois sistemas. Observando esse detalhe, como correção, esse mesmo autor, fazendo uso da expressão "transferência de Calor", ainda é de nosso entendimento que essa fonte é a que aborda sobre Temperatura com maior clareza.
\end{abstract}

Dando significado à Entropia e à Temperatura, vamos apresentar uma reflexão sobre o produto TS na equação de Helmholtz.

Como sabemos, a Entropia está associada à limitação da conversão de Calor em Trabalho. Nesse sentido, o produto TS é uma energia que representa o quantitativo da energia que não poderá estar disponível para realizar Trabalho, ou seja, é uma energia que não está mais disponível (útil). Com isso, TS é uma energia que deve ser subtraída da Energia Interna para garantir a validade da Segunda Lei da Termodinâmica. Todavia, o produto TS só poderá ter o significado de Calor quando o sistema estiver em transformação. Com isso, Calor não pode estar na relação TS, porque Calor é uma Função de Transformação, enquanto $U$ e TS são Variáveis de Estado.

O significado de $P V$ pode ser verificado na mesma lógica em que TS foi abordado. Com base em Correia e Oliveira (2019), podemos identificar uma característica notável da Função Helmholtz, com característica de Variável de Estado, que a difere de outras Funções Potenciais. Enquanto a Energia Interna é uma Função Potencial, que possui sua interpretação física no próprio Sistema, a Função Potencial de Helmholtz é a energia útil, descontando a quantidade $T S$ da Energia Interna $U$. A parcela de energia que foi retirada, depois de restabelecido o equilíbrio, é representada pelo produto TS. Logo, a Energia Livre de Helmholtz (F) é a máxima energia que pode ser retirada da Energia Interna de um sistema para ser convertida em trabalho.

Entretanto, como a Energia Livre de Helmholtz é uma função potencial, apenas a sua variação pode ser obtida em laboratório. Assim, apesar da existência real da expressão matemática da Função de Helmholtz $(F=U-T S)$, os livros costumam apresentar essa relação matemática, mas têm ignorado a sua definição, focando os seus esforços na definição da 
Variação da Energia Livre de Helmholtz. Sendo assim, observando apenas o tratamento dado pelos livros pesquisados, a definição da Energia Livre de Helmholtz, continua sendo uma incógnita. Todavia, conforme já apresentamos, acreditamos que o conhecimento de uma definição com precisão é indispensável, mesmo que essa grandeza não aparente ser tão aplicável, pois pode abrir novos horizontes tanto na formação quanto no labor de um Físico.

\section{Representação Matemática da Função de Helmholtz}

Além da definição das partes que compõe uma equação, acreditamos que, para uma real compreensão dessa equação, uma dedução matemática pode ser muito útil. Sendo assim, apresentaremos nesta seção uma forma de determinar a expressão matemática da Energia Livre de Helmholtz, bem como sua variação, o seu significado, sua conservação e o que essa consideração significa, bem como os significados e condições da sua maximização e minimização.

Segundo Correia e Oliveira (2018), a Primeira Lei da Termodinâmica garante que existe uma função característica do sistema que depende apenas do estado inicial e final de um sistema.” (2018, p.210). Logo, partindo dessa função característica temos que:

$$
d U=d^{\prime} Q-d^{\prime} W
$$

Sabemos que o Calor pode ser escrito como:

$$
d^{\prime} Q=T d S
$$

Enquanto que o trabalho, devido apenas a variação do volume, pode ser escrito da seguinte forma:

$$
d^{\prime} W=P d V
$$

Portanto, substituindo (2) e (3) em (1), temos:

$$
d U=T d S-P d V
$$

Definindo o produto $T S$ e diferenciando esse produto, temos:

$$
d(T S)=T d S+S d T
$$

Rearranjando a equação anterior:

$$
T d S=d(T S)-S d T
$$

Revista RBBA $\mid$ Revista Binacional Brasil Argentina 
Definindo o produto $P V$ e diferenciando-o:

$$
d(P V)=P d V+V d P
$$

Rearranjando a equação anterior:

$$
P d V=d(P V)-V d P
$$

Substituindo (6) e (8) em (4):

$$
d U=[d(T S)-S d T]-[d(P V)-V d P]
$$

Ou ainda:

$$
d U=d(T S)-S d T-d(P V)+V d P
$$

Considerando que houve uma transformação isocórica (volume constante) temos:

$$
d U=d(T S)-S d T-V d P+V d P
$$

Logo:

$$
d U=d(T S)-S d T
$$

ou:

$$
d U-d(T S)=-S d T
$$

Como a subtração das derivadas é a derivada da subtração:

$$
d(U-T S)=-S d T
$$

A Função de Helmholtz é uma nova propriedade do sistema definida pela relação matemática:

$$
F=U-T S
$$

Substituindo (15) em (14):

$$
d(F)=-S d T
$$

Logo, quando tratamos de uma transformação isocórica, "a Variação da Função de Helmholtz corresponde à energia, na forma de Calor, que não pode ser convertida em Trabalho".

No caso de uma transformação que além de isocórica, também for isotérmica, temos:

$$
d(F)=0
$$

\begin{tabular}{l|l} 
Revista RBBA & Revista Binacional Brasil Argentina
\end{tabular} 
Quando a Temperatura for constante, da equação (17) podemos concluir que a Função de Helmholtz se conserva. Encontramos uma condição de Conservação do Potencial de Helmholtz, para o caso em que a Temperatura não varia.

Da equação (15) poderemos concluir que a Energia Livre de Helmholtz é máxima na condição ideal em que a Entropia $(S)$ é mínima. Ou seja, a Energia Livre de Helmholtz é máxima quando a Entropia for nula ou quando a Temperatura do sistema tender ao Zero Absoluto. Nessa situação, toda Energia Interna $(U)$ do sistema poderia ser convertida integralmente em Trabalho, caso o sistema seja hipoteticamente destruído. Nessas circunstâncias, não haveria perda de energia condicionada pela Entropia. Ou ainda, toda Energia Interna seria considerada útil, uma vez que o produto TS seria nulo.

A equação (15) permite analisar quando a Energia Livre de Helmholtz é mínima. Isso ocorreria quando toda a Energia Interna do sistema fosse "perdida" devido ao aumento da Entropia. Sendo assim, teríamos um sistema com Entropia máxima. Ou seja, a grandeza $U$ teria o mesmo valor numérico do produto TS. Se esse sistema fosse "destruído", nenhuma energia poderia ser convertida em Trabalho. Mesmo assim, alcançaria um valor experimental, pois, se a Entropia fosse máxima, o sistema alcançaria o Equilíbrio Termodinâmico, pois a Energia Livre de Helmholtz passaria a ser constante e igual a zero.

\section{Materiais e Métodos}

A Energia Livre de Helmholtz é uma Função de Estado que faz parte das Funções Potenciais da Termodinâmica Clássica. E para embasar a nossa análise das definições da Energia Livre de Helmholtz apresentadas nas fontes pesquisadas, utilizamos uma metodologia semelhante à utilizada por Correia e Oliveira (2019), além da afirmação de Pádua et al (2008, p.58):

Quase todas as aproximações estabelecidas para a Termodinâmica Clássica seguem uma das duas alternativas: a aproximação histórica, que faz um estreito paralelo entre a evolução cronológica dos conceitos corretos e dos falsos juízos e, a aproximação postulatória, na qual são formulados postulados que são demonstrados "a priori", mas que podem ter suas veracidades confirmadas "a posteriori”.

Sendo assim, inspirados nessas duas aproximações (histórica e postulatória), analisamos as fontes pesquisadas, em busca da definição da Energia Livre de Helmholtz e da 
sua variação, bem como da discussão do seu máximo, do seu mínimo e da sua conservação, balizada pelo Silogismo de Aristóteles.

Pesquisamos quinze livros publicados entre 1971 e 2010, a saber: Costa (1971a); Sears e Salinger (1979); Zemansky (1978); Savi e Colucci (2010); Stowe (2007); Borgnakke, Richard e Wylen (1996); Callen (1985); Kittel e Kroemer (2000); Moran e Shapiro (2006); Oliveira (2005); Smith, Ness e Abbott (2007); Macedo e Luiz (1976); Schroeder (1999); Pádua e Pádua (2006) e Levenspiel (1999).

Além desses livros, pesquisamos em três sites, em um dicionário e uma enciclopédia.

\section{Resultados e Discussão}

\subsection{Investigação das Definições em Livros Didáticos}

O livro Termodinâmica Amistosa Para Engenheiros faz menção à Função de Gibbs, que é outro potencial termodinâmico. Todavia, não comenta sobre o Potencial de Helmholtz.

Sears e Salinger (1979) apresentam primeiro a relação $W_{T} \leq\left(F_{1}-F_{2}\right)$ para indicar que a energia disponível para realizar trabalho é, no máximo, equivalente ao negativo da variação da Função de Helmholtz. Nas palavras dos autores:

O decréscimo na função de Helmholtz de um sistema estabelece um limite superior para o trabalho em qualquer processo entre dois estados de equilíbrio à mesma temperatura, e durante o qual há um fluxo de calor para o sistema, proveniente de um reservatório a esta mesma temperatura. Se o processo for reversível, a entropia total do sistema mais o reservatório será constante (SEARS; SALINGER, 1979, p.163).

Apesar de concordarmos com a afirmativa: "o decréscimo da Função de Helmholtz de um sistema estabelece um limite superior para o trabalho", uma vez que a variação na função de Helmholtz corresponde à energia, na forma de Calor, que não pode ser convertida em trabalho, acreditamos que para que isso ocorra não é necessária a existência de uma condição de Equilíbrio Termodinâmico à Temperatura constante, pois, na equação $d(F)=-S d T$, se a Temperatura for constante, a Função de Helmholtz também será constante. Ou seja, apesar da afirmativa continuar válida, pois, a condição de equilíbrio à Temperatura constante é um caso particular da relação $d(F)=-S d T$, acreditamos que para melhor compreensão do leitor, Sears e Salinger deveriam apresentar a discussão tratando-o como caso geral. Conforme já afirmamos 
anteriormente, não tem sentido físico o termo "Fluxo de Calor", pois, segundo o Teorema de Gauss (Divergência), o fluxo só existirá para grandezas vetoriais.

Logo após, explicam o motivo de utilizarem o termo "Função de Helmholtz" ao invés de "Energia Livre de Helmholtz". Além disso, apresentam uma justificativa para essa energia ser considerada "Livre", considerando que ela é livre por estar disponível para realização de Trabalho, conforme o texto abaixo:

Porque seu decréscimo é igual à máxima energia que pode ser "liberada" em um processo e tornada disponível para trabalho, a grandeza $F$ é, algumas vezes, denominada a energia livre de um sistema. Entretanto, como o mesmo termo é aplicado também a outra propriedade a ser definida em breve, usaremos o termo "função de Helmholtz" para evitar confusão. (SEARS; SALINGER, 1979, p.163).

E ainda afirmam:

Note-se, entretanto, que embora o decréscimo na função de Helmholtz de um sistema seja igual ao trabalho máximo que pode ser obtido sob as condições acima, a energia convertida em trabalho e fornecida somente em parte pelo sistema, o restante provindo do calor retirado de um reservatório de calor. (SEARS; SALINGER, 1979, p.163).

Sears e Salinger justificam a origem da energia que será convertida em Trabalho. Todavia a redação dificulta a compreensão quando afirmam que nem toda a energia que será convertida em Trabalho será fornecida pelo sistema, que parte dela será retirada de um “Reservatório de Calor". O que, novamente, não tem significado físico, devido ao fato de Calor ser uma Função de Transformação e não pode ser fornecida nem retirada de um sistema.

E finalizam:

O decréscimo na função de Helmholtz estabelece, então, um limite superior para o trabalho "não-PdV" em um processo à temperatura e volume constantes. Se o processo for reversível este trabalho será igual ao decréscimo na função de Helmholtz. (SEARS; SALINGER, 1979; p.163).

Aqui foi definida a variação da Função de Helmholtz. Entretanto afirmam que a Temperatura é constante. Julgamos que essa informação deveria vir a posteriori, quando fosse abordada a situação em que a Função de Helmholtz não variasse.

Savi e Colucci (2010) apresentam a Energia Livre de Helmholtz, como: 
A função $F(T, V)$ é chamada de energia livre de Helmholtz. Essa é a energia total necessária para se criar o sistema, menos o calor que se pode tomar gratuitamente do meio à temperatura T. Esse calor é $T \Delta S=T S$, onde $S$ é a entropia final do sistema; quanto maior for a entropia de um sistema, maior a quantidade de energia que pode entrar na forma de calor. (SAVI, 2010, p. $101)$.

A afirmativa "é a energia total necessária para se criar o sistema, menos o calor que se pode tomar gratuitamente do meio à temperatura $T$ ' indica que a Energia Livre de Helmholtz está associada à criação de um sistema que possui uma energia que flui espontaneamente para o seu interior. Todavia, a relação $F=U-T S$ não faz menção à criação de sistema, pois se fossemos criar um sistema, a menos que ele seja criado no vácuo, seria necessário calcular pela Entalpia e não pela Energia Interna. E, mesmo se a transformação ocorresse no vácuo, o termo $T S$ não pode ser associado a uma energia que entra gratuitamente no sistema, devido à Entropia, tornando inutilizável parte da energia do sistema. Quando afirmam que "calor é $T \Delta S=T S "$, os autores criam um problema, pois, $T S$ é uma Variável de Estado, logo não pode ser associado ao Calor.

E ainda destacam:

Muitos processos importantes ocorrem isotermicamente e nesses casos a energia livre de Helmholtz é a variável adequada ou conveniente em termos de energia. Para justificar essa afirmação, devemos mostrar como a variação da função F está relacionada ao trabalho realizado sobre o sistema. (SAVI, 2010, p. 101).

Já Oliveira (2005) procura definir a matematicamente a Função de Helmholtz:

O uso da transformação de Legendre nos permite obter outras representações equivalentes da relação fundamental. Em outros termos, ela permite que se use outras grandezas termodinâmicas como variáveis independentes. Vamos, inicialmente fazer a mudança $(S, V, N) \rightarrow(T, V, N)$. Nessa nova representação, a relação fundamental é dada pelo potencial termodinâmico denominado Energia Livre de Helmholtz $F(T, V, N)$, definido pela transformada de Legendre

$$
F(T)=\min _{S}\{U(S)-T S\}
$$

que deve ser entendida como segue. Fixado um determinado valor da temperatura, variamos a entropia até encontrar o mínimo da expressão entre chaves, isto é, até encontra a menor diferença entre $U(S)$ e TS. Como $U(S)$ é 
diferenciável, o mínimo ocorre quando $U^{\prime}(S)=T$. Invertendo essa equação, obtemos $S(T)$ que, substituído em (4.31), dá

$$
F(T)=U(S)-T S
$$

(OLIVEIRA, 2005, p.58).

Apesar de não ter apresentado uma definição teórica com uma evolução cronológica de ideias, julgamos ser válida esta definição postulatória. Todavia, não julgamos suficiente o método didático nem ao apresentar uma definição que foque "apenas" na relação matemática nem ao apresentar uma forma de chegar até essa relação. Logo após apresentar essa definição, Oliveira (2005) explica como construir as Transformadas de Legendre para obter a equação que fornece a Energia Livre de Helmholtz.

A partir de (4.32) obtemos $F^{\prime}(T)=-S$. Portanto as derivadas $T(S)=\frac{\partial U}{\partial S}$ e $S(T)=-\frac{\partial F}{\partial T}$ formam um par de funções inversas, como pode ser visto na figura 4.1. Esse resultado fornece uma maneira de construir a Transformada de Legendre: (a) inicialmente determinamos a derivadas $T(S)$ de $U$ relativamente a $S$; (b) invertemos $T(S)$ para obter $S(T)$ que é a derivada de $-F$ relativamente a $T$; (c) a integral $S(T)$ fornece $F(T)$ com sinal contrário a menos de uma constante (OLIVEIRA, 2005, p. 59).

Após essas considerações, Oliveira passa a considerar o Potencial Químico nas equações, abrindo o sistema. Por outro lado, o estudo da Energia Livre de Helmholtz para sistemas abertos não faz parte do escopo deste artigo.

Zemansky (1978, p.254) apresenta em seu livro uma definição para a variação da Função de Helmholtz para considerar, em seguida, as condições para que ela não varia:

Por conseguinte, a variação da função de Helmholtz durante um processo isotérmico reversível é igual ao trabalho realizado sobre o sistema. (2) Para um processo reversível, isotérmico e isocórico,

$$
\begin{aligned}
& d F=0, \\
& \text { e } F=\text { constante }
\end{aligned}
$$

A equação (16) sugere que a variação da Função de Helmholtz fornece uma energia térmica na forma de Calor. Logo, essa variação pode até ser equivalente ao Trabalho realizado pelo sistema, mas, não é exatamente igual à energia disponível na forma de Trabalho. Outra 
observação é que Zemansky denomina o processo de isocórico apenas quando a Função de Helmholtz for constante e denomina de transformação isotérmica tanto na sua variação quanto na sua conservação. O que destoou dos outros livros analisados. Em seguida apresenta as áreas de conhecimento em que a Função de Helmholtz é particularmente útil.

Estas propriedades são de interesse em química e resultam úteis ao considerar-se as reações químicas que ocorrem isotérmica e isocoricamente. Entretanto, a importância principal da função de Helmholtz está na mecânica estatística, onde está intimamente associada à função partição $\mathrm{Z}$ definida pela equação

$$
Z=\Sigma g_{i} e^{-e_{i} / k T}, \quad(\text { ZEMANSKY, p.254, 1978). }
$$

Logo, a Função de Helmholtz não é útil apenas na Termodinâmica Clássica e na Termoquímica para estudo de sistemas em equilíbrio. Ela está associada, também, à Mecânica Estatística. Sendo assim, tem um campo de aplicação muito extenso.

Borgnakke, Richard e Wylen (1995) apresentam em seu livro a expressão matemática da Função de Helmholtz e, além disso, afirmam que essa função é uma propriedade termodinâmica que depende da massa:

A quantidade (U-TS) é uma propriedade termodinâmica de uma substância e é chamada de função de Helmholtz. É uma propriedade extensiva e a designamos pelo símbolo A. Assim

$$
\begin{aligned}
& A=U-T S \\
& a=u-T S
\end{aligned}
$$

(WYLEN; RICHARD; BORGNAKKE, 1995, p.234)

Em seguida os autores apresentam uma expressão para a obtenção do Trabalho reversível numericamente igual à soma das energias iniciais do sistema, menos a soma das energias finais, de uma forma bem distinta das outras fontes pesquisadas:

Portanto quando um sistema sofre uma mudança de estado, enquanto está em equilíbrio térmico com o meio, o trabalho reversível é dado pela relação

$$
{ }_{1} w_{2}^{\text {rev }}=\left(a_{1}+\frac{V_{1}^{2}}{2}+g Z_{1}\right)-\left(a_{2}+\frac{V_{2}^{2}}{2}+g Z_{2}\right)
$$


Nos casos em que as variações de energia cinética e potencial não são significativas, esta equação se reduz a

$$
{ }_{1} w_{2}^{r e v}=A_{1}-A_{2}=m\left(a_{1}-a_{2}\right)
$$

(WYLEN; RICHARD; BORGNAKKE, 1995, p.234)

Dessa forma, os autores apresentaram uma expressão matemática para Energia Livre de Helmholtz, afirmando que é uma grandeza extensiva, obtiveram uma expressão para o trabalho reversível, mas, não definiram a Energia Livre de Helmholtz.

Callen (1985, p.159) afirma que:

Assim, o trabalho entregue em um processo reversível, por um sistema em contato com um reservatório térmico, é igual ao decréscimo do potencial Helmholtz do sistema. O potencial de Helmholtz é frequentemente chamado de "energia livre" de Helmholtz, embora o termo trabalho disponível à temperatura constante esteja menos sujeito a erros de interpretação. (Tradução nossa).

Logo, Callen apresenta uma definição para a variação do Potencial de Helmholtz, mas não apresenta uma definição para o potencial propriamente dito. Além disso, acreditamos que a variação do Potencial de Helmholtz é numericamente igual à energia do sistema, na forma de Calor, que não está mais disponível para a realização de Trabalho.

Costa (1971) apresenta a relação matemática para a Energia Livre de Helmholtz a partir das Transformadas de Legendre: "a energia livre ou potencial termodinâmico à volume constante é uma função potencial do tipo $F=\varphi_{3}(T, v)$." (COSTA, 1971a, p. 95). Logo, para Costa, apenas o volume precisa ser constante para obtermos a função de Helmholtz. Em seguida Costa apresenta a relação matemática que fornece a variação da Energia Livre de Helmholtz:

$$
\begin{gathered}
d U=T \cdot d S-A p \cdot d v \\
T \cdot d S=d(T . S)-S \cdot d T
\end{gathered}
$$

Obtemos:

$$
d U-d(T . S)=-S \cdot d T-A p \cdot d v
$$


E como a diferença entre duas grandezas de estado também é uma função do estado considerado, fazendo:

$$
d F=d U-d(T . S)
$$

Podemos dizer que:

$$
d F^{\prime}=-S \cdot d T-A p \cdot d v
$$

É a diferencial total exata de F. Donde as propriedades que seguem:

$$
\begin{gathered}
\left(\frac{\partial F}{\partial T}\right)_{v}=-S \\
\left(\frac{\partial F}{\partial v}\right)_{T}=-A p \\
\left(\frac{\partial S}{\partial v}\right)_{T}=A\left(\frac{\partial p}{\partial T}\right)_{v}
\end{gathered}
$$

O nome dado a Energia Livre de Helmholtz vem da sua interpretação física. (COSTA, 1971a, p. 96).

Todavia, Costa (1971) havia afirmado anteriormente que "a energia livre ou potencial termodinâmico à volume constante é uma função potencial do tipo $F=\varphi_{3}(T, v)$. (COSTA, 1971a, p. 95). Logo, o termo “dv", da equação " $d F=-S \cdot d T-A p . d v$ ” é igual a zero, pois o volume é constante, de modo que a variação da Função de Helmholtz deveria estar na seguinte forma: ، $d F=-S . d T$ ” e ainda apresenta uma definição para a Energia Livre de Helmholtz quando a Temperatura for constante, a saber:

Assim, para uma transformação isotérmica, podemos escrever que:

$$
d F=-A p \cdot d v=-A \cdot d L
$$

Isto é: "a variação da energia livre apresentada por um sistema que evolui isotermicamente é igual ao trabalho mecânico absoluto que entra em jogo com o mesmo, durante a transformação considerada (com o sinal contrário em vista da convenção adotada para o trabalho)". (COSTA, 1971a, p. 96).

Podemos notar que novamente foi negligenciada a afirmativa inicial de que o volume foi constante. Em um processo isotérmico, teríamos $d F=0$. Costa continua afirmando que: "A energia interna é constituída, portanto, de duas parcelas, uma ' $F$ ', que numa expansão térmica pode ser transformada integralmente em trabalho mecânico, e outra ' $T S$ ', que não é isotermicamente conversível em trabalho.” (COSTA, 1971a, p. 95). E ainda relata sobre uma limitação para a conversão da Energia Interna de um sistema em Trabalho devido ao termo 
"TS". Costa finaliza com a seguinte afirmativa: "do que ficou visto, fácil é compreender a razão das designações dadas a $F$ de energia livre e a $T S$ de energia ligada ou vinculada". Dessa forma, o termo " $T S$ " foi associado a uma energia vinculada ao sistema, enquanto " $F$ " é associado à energia do sistema que está livre, ou disponível.

Moran e Shapiro (2006) apresentam a equação da Função de Helmholtz, mas não a define:

As funções Helmholtz e Gibbs são propriedades porque cada uma é definida em termos de propriedades. Da inspeção das Eqs. 11.20 e 11.21, as unidades de $\boldsymbol{\psi}$ e g são as mesmas de u e h. Essas duas novas propriedades são introduzidas apenas porque contribuem para a presente discussão e, neste momento, não é necessário atribuir significado físico a elas. (MORAN; SHAPIRO, 1917, p.498, tradução nossa).

Kittel e Kroemer (2000) afirmam que:

$$
\begin{aligned}
& \text { A função } \\
& F=U-T S
\end{aligned}
$$

É chamado de energia livre de Helmholtz. Essa função desempenha o papel na física térmica à temperatura constante que a energia $U$ desempenha em processos mecânicos comuns, que sempre são entendidos como entropia constante, porque não são permitidas alterações internas de estado. A energia livre nos diz como equilibrar as demandas conflitantes de um sistema por energia mínima e máxima entropia. A energia livre de Helmholtz será o mínimo para um sistema $\boldsymbol{\delta}$ em contato térmico com um reservatório $\phi$, se o volume do sistema for constante. (KITTEL; KROEMER, 2000, p.68, tradução nossa).

A partir da definição apresentada por Kittel e Kroemer poderíamos concluir que a Energia Livre de Helmholtz sempre é mínima, mas, sabemos que o que geralmente ocorre é o oposto.

Schroeder (1999) apresenta a Energia Livre de Helmholtz da seguinte forma:

Frequentemente, no entanto, não estamos interessados na energia total necessária ou na energia total que pode ser recuperada. Se o ambiente é de temperatura constante, o sistema pode extrair calor desse ambiente gratuitamente. Portanto, tudo o que precisamos fornecer, para criar o sistema do nada, é qualquer trabalho adicional necessário. E se aniquilarmos o sistema, geralmente não podemos recuperar toda a sua energia como trabalho, porque temos que descartar sua entropia despejando um pouco de calor no ambiente.

Então, eu gostaria de introduzir mais duas quantidades úteis relacionadas à energia e análogo a H. Um é a energia livre de Helmholtz. (SCHROEDER, 1999, p.150, tradução nossa). 
Sabemos que as afirmativas "extrair calor desse ambiente" e "despejando um pouco de calor no ambiente" não têm significado físico, pois, o Calor não pode pertencer a um sistema para ser "extraído" ou "despejado". Schroeder ainda afirma: "Portanto, $F$ é a energia que deve ser fornecida como trabalho, se você estiver criando o sistema do nada". (SCHROEDER, 1999, p.150, tradução nossa). Entendemos que criar um "sistema do nada" contradiz os princípios da Termodinâmica, mas esse jargão é comumente utilizado.

Macedo e Luiz (1976) iniciam a discussão sobre a Energia Livre de Helmholtz ou Potencial Isocórico-Isotérmico, considerando que essa é uma Função de Estado e apresenta uma relação matemática para a Função de Helmholtz e para a sua variação, a saber:

A função de estado energia livre (energia livre de Helmholtz ou potencial isocórico-isotérmico) é definida pela relação:

$F=U-T S$.

Sob forma diferencial:

$d F=-S . d T-P . d w$,

onde $d w$ é o trabalho infinitesimal fornecido pelo sistema em processos quase-estáticos. (MACEDO; LUIZ, 1976, p.89).

Macedo e Luiz ainda apresentam o Trabalho na forma diferencial $d w$. Todavia, tal notação de diferencial exata só seria coerente se $w$ fosse uma variável de estado. Logo, ao invés da notação " $d w$ " os autores deveriam ter utilizado a notação de diferencial inexata para indicar um trabalho infinitesimal. Macedo e Luiz continuam o texto com a seguinte afirmação:

No caso de um gás que só fornece trabalho de expansão, $d w=p \cdot d v$ e então,

$d F=-S \cdot d T-p \cdot d V$.

Numa transformação finita isotérmica, a variação de energia livre mede o trabalho que, no máximo, pode ser extraído do sistema, com o sinal negativo:

$\Delta F=-w_{\max }$.

Como é claro esse trabalho é o que pode ser fornecido no processo isotérmico quase-estático. (MACEDO; LUIZ, 1976, p.89). 
Segundo Correia e José (2011), um sistema não cede nem recebe Trabalho. Macedo e Luiz consideram que em um processo isotérmico "a variação de energia livre mede o trabalho que, no máximo, pode ser extraído do sistema, com o sinal negativo”. Todavia, os mesmos autores consideraram o caso em que o gás sofre apenas expansão. Entretanto, a Energia Livre de Helmholtz o processo é para processos isocóricos. Considerando essa situação, a variação da Função de Helmholtz deveria ser nula.

Stowe apresenta a variação da Energia Livre de Helmholtz da seguinte forma: " $d F_{0} \leq 0 "$. Ou seja, para sistemas que interagem isotermicamente, a Segunda Lei da Termodinâmica exige que mudanças na Energia Livre de Helmholtz devem ser negativas, atingindo um mínimo de equilíbrio". (STOWE, 2007, p.169, tradução nossa). Concordamos com o fato da Segunda Lei da Termodinâmica garantir que a variação da Energia Livre de Helmholtz deve ser sempre menor ou igual a zero, pois, a Entropia de um sistema deve sempre aumentar. Todavia não há necessidade da transformação ser isotérmica. A transformação ser isocórica já é uma condição suficiente. Stowe (2007) ainda inclui o Potencial Químico na sua abordagem sobre a Energia Livre de Helmholtz. Porém, como afirmamos anteriormente, para tratar do Potencial Químico, o sistema deveria ser aberto.

Smith, Ness e Abbott (2007, p. 149), talvez por se tratar de um livro de Química, não apresenta uma definição para a Energia Livre de Helmholtz, apenas apresentam a equação da seguinte forma:

Todas as propriedades termodinâmicas primárias - $\underline{\mathrm{P}}, V, T, U$ e $S$ - estão presentes na eq. (6.1). Propriedades termodinâmicas adicionais somente aparecem por definição em relação a essas propriedades primárias. A entalpia, definida por conveniência no capítulo 2, é:

$H \equiv U+P V$

Duas propriedades adicionais, também definidas por conveniência, são a energia de Helmholtz e a energia de Gibbs:

$$
\begin{aligned}
& A \equiv U-T S \\
& G \equiv H-T S
\end{aligned}
$$

Pádua e Pádua (2006) abordam Potencial de Helmholtz utilizando exercícios de aplicação. Também apresentaram a equação da Função de Helmholtz relacionando-a à Transformada de Legendre da seguinte forma: "Representação funcional: $F(T, V, N)$.. A transformada de Legendre gera a seguinte Equação Fundamental: $F=U-T S$ " (PÁDUA; PÁDUA, 2006, p.260). Ou seja, apresentam a equação, mas não definem Energia Livre de Helmholtz nem a sua variação.

Revista RBBA Revista Binacional Brasil Argentina 
Magalhães, Zambrano e Sicezkowski (1973), no Dicionário de Física, apresentam a Energia Livre de Helmholtz "definida pela equação: $F=U-T S$. A combinação das propriedades termodinâmicas: energia interna $U$, temperatura absoluta $T$ e entropia $S$ dá uma propriedade $F$, que é a função de Helmholtz, também chamada função do trabalho." (MAGALHÃES; ZAMBRANO; SICEZKOWSKI, 1973, p. 286). Em seguida abordam a limitação de obtenção de energia ao qual a Energia Livre de Helmholtz está associada.

Pode-se utilizar somente uma parcela da energia interna, dada por $U-T S$, que foi denominada energia livre ou potencial isostérico. Nos processos isotérmicos reversíveis, o trabalho máximo obtido do sistema é dado pela diminuição da energia livre. Sendo a transformação monoterma, isotérmica e reversível, seu valor é constante, mas diminui no caso real (irreversível). (MAGALHÃES; ZAMBRANO; SICEZKOWSKI, 1973, p. 286).

A Energia Livre de Helmholtz não é toda a energia do sistema e a sua variação é negativa. Os autores ainda afirmam que, no caso específico em que a Temperatura é constante, o processo passa a ser reversível e o valor da Energia Livre de Helmholtz passa a ser constante. Entretanto, para o caso real o valor diminui. Apesar de concordarmos com o que foi apresentado pelo dicionário, faltou explicar ou justificar o motivo pelo qual a Energia Livre de Helmholtz sofre redução da sua intensidade. Além disso, apesar de terem proposto definirem Energia Livre de Helmholtz, foi discutida apenas a sua variação.

A enciclopédia Ciência Ilustrada apresenta a Energia Livre de Helmholtz juntamente com a Energia Livre de Gibbs. Faz um breve comentário sobre a importância desses potenciais e apresentam as suas relações matemáticas.

As diferenças entre certas funções termodinâmicas, como $U$-TS e $H$-TS, são muito importantes porque permitem calcular a que distância do equilíbrio determinado sistema termodinâmico se encontra. Por esse motivo, essas diferenças, que recebem o nome de potenciais termodinâmicos, são indicados por dois símbolos especiais:

$F=U-T S$

$G=H-T S$

F chamada a função de Helmholtz, e G função de Gibbs.

Logo, a enciclopédia relaciona esses dois potenciais termodinâmicos ao cálculo da "distância" que um determinado sistema está de seu estado de equilíbrio termodinâmico, reforçado na seguinte afirmação: 
Conhecida a energia potencial mecânica de um sistema físico, pode-se calcular o trabalho que esse sistema desenvolverá ao se deslocar em direção a uma configuração de equilíbrio: será igual à diferença entre as energias potenciais do estado inicial e do estado final. Da mesma forma, as funções de Gibbs e de Helmholtz permitem calcular a energia que um processo termodinâmico espontâneo pode fornecer ao exterior. É evidente a importância desses potenciais termodinâmicos no estudo das reações químicas.

Portanto, uma das principais aplicações da Energia Livre de Helmholtz, segundo essa enciclopédia, é no estudo de reações químicas, conforme foi destacado: "a energia livre de Helmholtz $(F)$ é útil no estudo de reações que ocorrem a volume constante". Apesar da enciclopédia "Ciência Ilustrada" indicar qual a importância da Energia Livre de Helmholtz e a sua condição, em nenhum momento foi apresentada nem a definição da Energia Livre de Helmholtz nem a definição da sua variação.

\subsection{Definição em Sites da Internet}

É de fácil constatação que o uso de computadores e celulares, com acesso à internet, para a pesquisa dos mais diversos assuntos, inclusive pesquisas no âmbito acadêmico, vêm se tornando cada dia mais popular. Por conta disso, fizemos uma busca por definições da Energia Livre de Helmholtz em sites.

Na Wikipedia encontramos uma definição da Energia Livre de Helmholtz bastante interessante, cuja abordagem associa à energia útil de um sistema:

Na termodinâmica, a energia livre de Helmholtz é uma grandeza que mensura a parcela de energia interna de um sistema possível de ser utilizada na forma de trabalho. É particularmente útil na compreensão e descrição de processos isotérmicos: à temperatura constante a variação da energia livre de Helmholtz encontra-se diretamente associada ao trabalho total realizado pelo sistema sobre sua vizinhança, ou seja, é a energia útil que sobra para uso depois que o sistema utilizou parte da energia interna para expandir as fronteiras do sistema $(-P d V)$ e redistribuir as moléculas nos diferentes níveis quânticos de energia $(T d S)$ para processos à temperatura e volume constante. ${ }^{\text {ii }}$

Apesar de ser uma abordagem bem interessante, quando é dito: "encontra-se diretamente associada ao trabalho total realizado pelo sistema sobre sua vizinhança, ou seja, é a energia útil que sobra para uso depois que o sistema utilizou parte da energia interna para 
expandir as fronteiras do sistema $(-P d V)$ ", está sendo considerado um processo em que o volume varia. Mas, como sabemos, e como é dito mais a frente no mesmo parágrafo do site, a Energia Livre de Helmholtz é aplicada apenas a sistemas cujo volume é constante. Logo após, esse mesmo site, apresenta a sua definição para Energia Livre de Helmholtz:

Dada a segunda lei da termodinâmica, o conceito deriva da verificação que nem toda a energia interna de um sistema é passível de produzir trabalho, visto que uma parcela dessa energia encontra-se diretamente associada à entropia do sistema. Sendo a parcela de energia associada à entropia determinável pelo produto da entropia $S$ do sistema pela sua temperatura $T$, tem-se que a energia livre de Helmholtz é corretamente definida pela expressão: $U-T S$

Mensura-se com a energia livre de Helmholtz à totalidade da parcela de energia interna passível de implicar trabalho, quer esta parcela de energia venha a implicar trabalho "útil" - o movimento desejado nas máquinas térmicas, a exemplo - quer esta venha a implicar trabalho associado à variação de volume do sistema frente à pressão ambiente - como aquele relacionado à expansão dos gases de descarga expelidos pelos automóveis, a exemplo.

Esta é uma das melhores definições encontradas nessa pesquisa. Apesar de praticamente apenas ler a equação e aplicar a Segunda Lei da Termodinâmica, o texto é de simples e direto. Todavia, no final do parágrafo, volta a relacionar a Energia Livre de Helmholtz ao Trabalho associado à variação de volume do sistema, que contradiz sua premissa, confundindo Energia Livre de Helmholtz com Energia Livre de Gibbs, sobretudo por ter mantido a pressão constante (ambiente).

Outro site pesquisado faz apenas uma simples leitura da equação ao tentar defini-la: "Energia Livre de Helmholtz (A) e a energia interna de um sistema menos a energia 'não disponível' $T S$.',iii

Todavia, esse mesmo site, no tópico "Energia de Gibbs (G) e de Helmholtz (A) (Parte II): Propensão para Reações”, apresenta a relação matemática da Energia Livre de Helmholtz e argumenta da seguinte maneira:

Onde A é a energia livre de Helmholtz, E é a energia interna, T é a temperatura e S é a entropia. A energia livre de Helmholtz é aplicada em processos a temperatura e volume constantes enquanto a de Gibbs é aplicada à temperatura e pressão constantes. Processos isotérmicos são comuns, porém a inexistência do trabalho (condição isovolumétrica) é uma restrição para a utilização da equação. ${ }^{\text {iv }}$ 
O site nem define a Energia Livre de Helmholtz nem a sua variação, apenas apresenta a relação matemática e uma condição necessária (volume constante) para que seja aplicada. Essa fonte pesquisada fez a escolha de um sistema criado do nada para não precisar explicar a origem da energia. Apesar de não ter dito, como a Entropia inicial é nula, o sistema foi criado no "nada".

\section{Considerações Finais}

Conforme apresentamos na seção 5, nenhum dos livros ou sites apresentou uma interpretação física precisa para Energia Livre de Helmholtz. Das categorias (argumentação histórica e argumentação postulatória) analisadas, a maioria dos autores optou pela segunda, apresentando a Energia Livre de Helmholtz como sendo uma construção matemática conveniente elaborada para destacar propriedades úteis à descrição do sistema. Nesse sentido, não foi dado destaque à abordagem teórica. Ou seja, em alguns casos, houve uma simples leitura da equação $F=U-T S$ e a partir dela foram obtidas propriedades com justificativas experimentais úteis em estudos futuros.

A equação matemática da Energia Livre de Helmholtz contém duas parcelas. Uma dessas parcelas é a Energia Interna $(U)$ do Sistema, enquanto que a outra parcela é dada pelo produto $T S$. Nesse caso, $T$ representa a Temperatura atual do sistema e $S$ representa a Entropia do sistema durante a observação, caso fosse possível medi-la. Contudo, não foi apresentado como chegar a tal relação por meio de aparatos experimentais. Algumas delas fornecem uma explicação teórica partindo de princípios físicos para chegar à relação matemática da Energia Livre de Helmholtz.

Entretanto, mesmo nos livros e sites analisados que buscavam definir a Energia Livre de Helmholtz, partindo de teorias físicas, percebemos ausência de argumentações lógicas. As fontes pesquisadas não respeitaram as premissas que elas mesmas propuseram, a exemplo dos casos em que os textos afirmaram que o processo se dá sem variação do volume, para mais a adiante entrarem em contradição e relacionarem a variação de tal processo a um trabalho de expansão, ou negaram as próprias premissas físicas, quando na elaboração da definição de Energia Livre de Helmholtz com base em uma visão equivocada sobre que o significado de Calor, Trabalho, bem como de outras grandezas termodinâmicas.

Em suma, nenhuma das fontes pesquisadas que definiu Energia Livre de Helmholtz com precisão e simplicidade. Portanto, apresentaremos aqui a nossa definição da Energia \begin{tabular}{l|l} 
Revista RBBA & Revista Binacional Brasil Argentina
\end{tabular} 
Livre de Helmholtz: a "Energia Livre de Helmholtz $(F)$ é uma grandeza que fornece a máxima energia que pode ser retirada da Energia Interna de um Sistema para ser convertida em Trabalho." Caso esse sistema seja "destruído", não poderemos recuperar, de forma "útil", toda a energia outrora possuía na forma de Energia Interna, já que parte dessa energia, conforme estabelece a Segunda Lei da Termodinâmica, fica indisponível. Sendo assim, a "Energia Livre de Helmholtz $(F)$ é a energia que um sistema pode liberar, de forma útil, a partir da sua Energia Interna $(U)$ ”, considerando que energia útil é a quantidade de energia disponível para realização de trabalho.

Embora essas definições façam referência ao "Trabalho" e à energia "útil", devemos alertar que a energia em forma de Trabalho só poderá tornar disponível quando for obtida a variação da Energia Livre de Helmholtz.

Quanto à definição da Variação da Energia Livre de Helmholtz, os livros e sites pesquisados também apresentaram definições não satisfatórias. Acreditamos que a melhor definição vista foi a apresentada na seção 3. Nela definimos a variação da Energia Livre de Helmholtz da seguinte forma: "a variação na função de Helmholtz, numa transformação isocórica, corresponde à energia, na forma de calor, que não pode ser convertida em Trabalho".

Identificamos falta de abordagem quanto ao máximo e ao mínimo do Potencial de Helmholtz e da falta de abordagem do seu significado físico, mas apresentamos nosso entendimento sobre tais questões no final da seção 3 .

Talvez as fontes pesquisadas não tivessem a pretensão de dar significado para Energia Livre de Helmholtz, mas de apresentar algumas de suas propriedades úteis, para aplicar a uma situação experimental e, dessa forma, justificar o seu estudo.

Em suma, é esperado que sejam encontradas dificuldades para definir qualquer grandeza potencial, justificando algumas limitações encontradas nas fontes pesquisadas, sobretudo quando o foco não é uma abordagem teórica e sim aplicável. Todavia, acreditamos que para uma real compreensão das grandezas da Física, uma compreensão teórica torna indispensável. 


\section{REFERÊNCIAS}

CALLEN, H. B. Thermodynamics and an Introduction to Thermostatistics, $2^{\mathrm{a}}$ Edition, Republic of Singapore: Editora John Wiley \& Sons, 1985.

Ciência Ilustrada, v. 10. São Paulo: Editora Abril Cultural, 1972.

CORREIA, J. J. Definições de Temperatura em Fontes didáticas. Revista Binacional BrasilArgentina: Diálogo entre as ciências, [S.1.], v. 6, n. 1, p. 201-220, out. 2017. ISSN 23161205. Disponível em: http://periodicos2.uesb.br/index.php/rbba/article/view/1520. Acesso em: 10 nov. 2019. Doi: https://doi.org/10.22481/rbba.v6i1.1520.

CORREIA, J. J.; OLIVEIRA, W. C. A Definição de Energia Interna e o Enunciado da Primeira Lei da Termodinâmica nos Livros Didáticos. Revista Binacional Brasil-Argentina: Diálogo entre as ciências, [S. 1.], v. 7, n. 2, p. 184-215, dez. 2018. ISSN 2316-1205. Disponível em: http://periodicos2.uesb.br/index.php/rbba/article/view/4647. Acesso em: 10 nov. 2019.

CORREIA, J. J.; OLIVEIRA, W. C. A Definição de 'Entalpia' em Livros Didáticos. Revista Binacional Brasil-Argentina: Diálogo entre as ciências, [S. 1.], v. 8, n. 1, p. 327-353, maio 2019. ISSN 2316-1205. Disponível em: http://periodicos.uesb.br/index.php/rbba/article/view/4912. Acesso em: Acesso em: 10 nov. 2019.

CORREIA, J. J.; ORTIGOZA, L. V. O Conceito de Conservação de Energia em Livros Didáticos: uma análise histórico-didática. Revista Binacional Brasil-Argentina: Diálogo entre as ciências, [S. 1.], v. 4, n. 2, p. 91-103, set. 2017. ISSN 2316-1205. Disponível em: http://periodicos2.uesb.br/index.php/rbba/article/view/1472. Acesso em: 10 nov. 2019.

CORREIA, J.J.; MAGALHÃES, L. D. R. Obstáculos Epistemológicos na Transposição Didática do Calor. In: IX CÓLÓQUIO DO MUSEU PEDAGÓGICO. (ISSN 2175-5493), Vitória da Conquista (BA), Anais. Vitória da Conquista. MUSEU PEDAGÓGICO CASA PADRE PALMEIRA. 2009. v. 8, n. 1. p. 727-740, 2009. Disponível em: http://periodicos.uesb.br/index.php/cmp/article/viewFile/3574/3265. Acesso em: 10 nov. 2019.

CORREIA, J.J.; MAGALHÃES, L. D. R.; LIMA, L. S. Obstáculos Epistemológicos e o Conceito de Calor. Scientibus Série Ciências Físicas 04: 1-10, 2008. Disponível em: http://dfis.uefs.br/sitientibus/vol4/Jornandes-Main-SPSS2008.pdf. Acesso em: 10 nov. 2019.

CORREIA, J.J; JOSÉ, W. D. O conceito de Entropia e as Leis da Termodinâmica em Livros Didáticos de Física. In. X CÓLÓQUIO DO MUSEU PEDAGÓGICO (ISSN 2175-5493), vol. 10, $\mathrm{N}^{\mathrm{o}}$ 1, 2013. Vitória da Conquista (BA). MUSEU PEDAGÓGICO CASA PADRE PALMEIRA. 2013. Anais. Vitória da Conquista Disponível em: http://periodicos.uesb.br/index.php/cmp/article/viewFile/3008/2717. Acesso em: 10 nov. 2019.

CORREIA, J.J; JOSÉ, W. D. O Conceito de Trabalho de uma Força em Livros Didáticos, In: IX COLÓQUIO DO MUSEU PEDAGÓGICO (ISSN 2175-5493), Vitória da Conquista (BA), 
Anais. Vitória da Conquista. MUSEU PEDAGÓGICO CASA PADRE PALMEIRA. 2011. v. 9, n. 1, p. 727-740, 2011. Disponível em: http://periodicos.uesb.br/index.php/cmp/article/viewFile/2663/2330. Acesso em: 10 nov. 2019.

COSTA, E. C. Física Industrial - Enciclopédia Técnica Universal. Tomo I, Termodinâmica, I Parte. Porto Alegre: Globo, 1971a.

GILBERT, A. Origens Históricas da Física. Moderna: Introdução Abreviada. Fundação Calouste Gulbenkian. Lisboa. Portugal, 1982.

KITTEL, C., KROEMER, H. Thermal Physics. $2^{\text {a }}$ Edition, USA: W.H Freeman \& Company, $21^{a}$ printing in USA, 2000.

LEITE, M. S. S. C. P.; ALMEIDA, M. J. B. M. Compreensão de termos científicos no discurso da ciência. Rev. Bras. Ens. Fís., São Paulo, v. 23, n. 4, p. 458-470, Dec. 2001. Available from http://www.scielo.br/scielo.php?script=sci_arttext\&pid=S180611172001000400011\&lng=en \&nrm=iso. access on 12 Oct. 2019.

LEVENSPIEL, O. Termodinâmica Amistosa para Engenheiros, 38 ${ }^{a}$ Edição, São Paulo: Editora Edgard Blucher Ltda, 1999.

MACEDO, H.; LUIZ, A. M. Problemas de Termodinâmica Básica. São Paulo: Editora Edgard Blücher, 1976.

MAGALHÃES, A.; ZAMBRANO, R. S.; SIECZKOWSKI, R. P. S. Dicionário de Física. Porto Alegre: Editora Globo, 1973.

MÁXIMO, A.; ALVAREnGA B. Curso de Física, volume 2. $1^{\text {a }}$ Edição. São Paulo. EditoraScipione: 2011.

MORAN, M. J.; SHAPIRO, H. N. Fundamentals of Engineering Thermodynamics, $5^{\text {a }}$ Edition, USA: Editora John Wiley \& Sons Inc., 2006.

OLIVEIRA, M. J. Termodinâmica. São Paulo: Editora Livraria da Física, 2005.

PÁDUA, A. B. et al. Termodinâmica clássica ou termodinâmica do equilíbrio: aspectos conceituais básicos. Semina: Ciências Exatas e da Terra, Londrina, v. 29, n. 1, p. 57-84, jan./jun. 2008.

SAVI, A. A.; COLUCCI, C. C. Termodinâmica, Coleção Formação de Professores em Física - EAD Vol. 10, Maringá: Editora Eduem, 2010.

SCHROEDER, D. V. Introduction to Thermal Physics. $1^{\text {a }}$ Edition, USA: Editora Addison Wesley Longman, 1999. 
SEARS, F. W.; SAlingER, G. L. Termodinâmica, Teoria Cinética e Termodinâmica Estatística. $3^{\text {a }}$ Edição, Rio de Janeiro: Editora Guanabara Dois S.A, 1979.

SMITH, J. M., NESS, H. C., ABBOTT, M. M. Introdução à Termodinâmica da Engenharia Química, 7ª Edição, Rio de Janeiro: LTC, 2007.

STOWE, K. An Introduction to Thermodynamics and Statistical Mechanics. $2^{\mathrm{a}}$ ed. Cambridge: Cambridge University Press, 2007.

WYLEN, G. J. V.; SONNTAG, R. E.; BORGNAKKE, C. Fundamentos da termodinâmica clássica. 4. ed. Tradução de Euryale de Jesus Zerbini e Ricardo Santilli Ekman Simões. São Paulo: Editora Edgard Blücher, 1995.

ZEMANSKY, M. W. Calor e Termodinâmica. $5^{\text {a }}$ Edição, Rio de Janeiro: Editora Guanabara Dois S.A, 1978.

\section{Notas}

iDisponível em https://www.researchgate.net/post/Can_anyone_please_explain_why_the_Helmholtz_ free_energy_is_called_free. Acesso em: 11 nov. 2019.

ii Disponível em https://pt.wikipedia.org/wiki/Energia_livre_de_Helmholtz. Acesso em: 10 nov. 2019.

iii Disponível em http://www.cienciadosmateriais.org/index.php?acao=exibir\&cap=15\&top=268. Acesso em: 10 nov. 2019.

iv Disponível em http://www.cienciadosmateriais.org/index.php?acao=exibir\&cap=15\&top=273. Acesso em: 10 nov. 2019.

\section{Sobre os autores}

Jornandes Jesús Correia é Doutor em Física pela Universidade Federal de São Carlos (UFSCar), Mestre em Meteorologia Agrícola, pela Universidade Federal de Viçosa (UFV), na Área de Radiação Solar e Terrestre. na Área de Física Atômica e Molecular. Licenciado e Bacharel em Física pela Universidade Federal da Bahia (UFBA) tem Pós-Graduação Lato Sensu em Matemática Superior pela Fundação Educacional Severino Sombra. Desenvolve trabalhos na Área de Ensino de Física no Grupo de Pesquisa DIDÁTICA DAS CIÊNCIAS EXPERIMENTAIS E DA MATEMÁTICA (GDICEM), junto ao Museu Pedagógico Casa Padre Palmeira (MP). É Professor Pleno e Decano do Departamento de Ciências Exatas e Tecnológicas (DCET), Campus de Vitória da Conquista, da Universidade Estadual do Sudoeste da Bahia (UESB).

Gabriel Fonseca Guimarães é Estudante de graduação do curso de Licenciatura em Física, da Universidade Estadual do Sudoeste da Bahia, Campus de Vitória da Conquista. 